TTR

Traduction, terminologie, rédaction

\title{
La relecture unilingue : une procédure de révision de traduction rapide, fonctionnelle, mais déloyale
}

\section{Isabelle S. Robert}

Volume 27, numéro 1, 1er semestre 2014

Lecture et traduction

Reading and Translation

URI : https://id.erudit.org/iderudit/1037120ar

DOI : https://doi.org/10.7202/1037120ar

Aller au sommaire du numéro

\section{Éditeur(s)}

Association canadienne de traductologie

ISSN

0835-8443 (imprimé)

1708-2188 (numérique)

Découvrir la revue

Citer cet article

Robert, I. S. (2014). La relecture unilingue : une procédure de révision de traduction rapide, fonctionnelle, mais déloyale. TTR, 27(1), 95-122.

https://doi.org/10.7202/1037120ar

\section{Résumé de l'article}

Depuis 2006, les agences de traduction européennes qui souhaitent travailler conformément à la norme EN 15038 sur les services de traduction doivent intégrer la révision dans leur processus. Toutefois, la norme n'est pas claire quant à la manière dont cette révision doit être effectuée et en particulier quant à la nécessité de comparer les textes source et cible durant la révision. Ce constat est à la base d'une étude en cours devant déterminer l'impact de la procédure de révision sur le produit et le processus de révision. Dans la présente contribution, une procédure de révision non comparative, la relecture unilingue, sera comparée à trois autres procédures : la relecture bilingue et deux relectures dites « doubles ", à savoir la relecture bilingue suivie d'une relecture unilingue et la relecture unilingue suivie d'une relecture bilingue. L'objectif est de déterminer si la relecture unilingue est efficace et, le cas échéant, dans quelles conditions et par rapport à quelle autre procédure. Pour ce faire, les scores de seize réviseurs professionnels ayant révisé quatre textes cibles comparables chaque fois selon une procédure différente ont été comparés en ce qui concerne la qualité de la révision (produit), la détection d'erreurs et la durée du processus de révision (processus). Une analyse de la variance (ANOVA) a révélé que la relecture unilingue n'est pas significativement moins efficace, en termes de qualité et de potentiel de détection d'erreurs, qu'une relecture bilingue, mais qu'elle ne prend pas non plus significativement moins de temps. En revanche, si la relecture unilingue est significativement moins efficace que les relectures doubles, elle prend auss significativement moins de temps.
Ce document est protégé par la loi sur le droit d'auteur. L’utilisation des services d’Érudit (y compris la reproduction) est assujettie à sa politique d'utilisation que vous pouvez consulter en ligne.

https://apropos.erudit.org/fr/usagers/politique-dutilisation/ 


\title{
La relecture unilingue : une procédure de révision de traduction rapide, fonctionnelle, mais déloyale
}

\author{
Isabelle S. Robert \\ Universiteit Antwerpen - Groupe de recherche TricS
}

\section{Résumé}

Depuis 2006, les agences de traduction européennes qui souhaitent travailler conformément à la norme EN 15038 sur les services de traduction doivent intégrer la révision dans leur processus. Toutefois, la norme n'est pas claire quant à la manière dont cette révision doit être effectuée et en particulier quant à la nécessité de comparer les textes source et cible durant la révision. Ce constat est à la base d'une étude en cours devant déterminer l'impact de la procédure de révision sur le produit et le processus de révision. Dans la présente contribution, une procédure de révision non comparative, la relecture unilingue, sera comparée à trois autres procédures: la relecture bilingue et deux relectures dites «doubles", à savoir la relecture bilingue suivie d'une relecture unilingue et la relecture unilingue suivie d'une relecture bilingue. L'objectif est de déterminer si la relecture unilingue est efficace et, le cas échéant, dans quelles conditions et par rapport à quelle autre procédure. Pour ce faire, les scores de seize réviseurs professionnels ayant révisé quatre textes cibles comparables chaque fois selon une procédure différente ont été comparés en ce qui concerne la qualité de la révision (produit), la détection d'erreurs et la durée du processus de révision (processus). Une analyse de la variance (ANOVA) a révélé que la relecture unilingue n'est pas significativement moins efficace, en termes de qualité et de potentiel de détection d'erreurs, qu'une relecture bilingue, mais qu'elle ne prend pas non plus significativement moins de temps. En revanche, si la relecture unilingue est significativement moins efficace que les relectures doubles, elle prend aussi significativement moins de temps.

Mots-clés: révision de traduction, procédures de révision, qualité de la révision, étude de processus, analyse statistique

\section{Abstract}

Since 2006, European translation agencies willing to work according to the EN 15038 standard on translation services have had to include revision in their process. However, the standard is not clear as to how revision has to be conducted and in particular, to the necessity of comparing source 
and target texts during the revision process. This observation lies at the basis of an ongoing research project on the impact of revision procedure on revision product and process. In this paper, a noncomparative revision procedure, named monolingual revision, will be compared to three comparative procedures: bilingual revision and two "double revisions," i.e., a bilingual revision followed by a monolingual revision and a monolingual revision followed by a bilingual revision. The aim is to determine whether monolingual revision is efficient and if so, in what conditions and compared to which procedures. In the main study, the scores of 16 professional revisers, who had to revise four comparable target texts using one of the four procedures each time, have been compared as to quality of the revision (product), error detection potential, and process duration (process). An analysis of the variance (ANOVA) has shown that monolingual revision is not significantly less efficient than bilingual revision as to quality and error detection potential, but it does not take significantly less time either. In contrast, monolingual revision is significantly less efficient than double revisions, but it does take significantly less time.

Keywords: translation revision, revision procedures, revision quality, process studies, statistical analysis

\section{Introduction}

Bien que la révision ait conquis sa place dans le champ traductologique depuis quelques années déjà, avec la publication d'une des premières thèses sur le sujet (Brunette, 1997) et des premiers manuels (Horguelin et Brunette, $1998^{1}$; Mossop, 2001), elle demeure un thème de recherche marginal, comparée à la masse de publications consacrées à d'autres champs de recherche traductologique. Les chercheurs qui se sont penchés sur l'activité révisante le confirment (Chakhachiro, 2005; Lee, 2006; Shih, 2006), de même que ceux qui en ont fait un de leurs domaines de prédilection:

It is very common in the translation industry that somebody else than the original translator revises the translation. [...] Revision has become a well-defined step in the process of producing translations. However, very little has been written on translation revision. (Künzli, 2005, p. 31)

Depuis le début des années 2000, on observe un intérêt marqué pour la pratique de la révision des traductions. Nous en voulons pour preuve la publication en 2001 du manuel

1. Une mise à jour est parue depuis lors: Horguelin et Pharand, 2009. 
de révision de Mossop et la part faite à la révision dans le numéro spécial (2000) de The Translator sur l'évaluation des traductions. Depuis, quelques communications ou articles éclairants sur le sujet ont fait avancer la discipline. Mais on réserve toujours à cette dernière la portion congrue, par rapport, par exemple, aux questions interculturelles en traduction, aux effets des technologies sur les pratiques ou à la formation des traducteurs. (Brunette, 2007, p. 2)

En Europe, l'importance de la révision a été confirmée en 2006 avec la publication de la norme européenne 15038 sur les «Services de traduction - Exigences requises pour la prestation du service» qui fait de cette activité une étape obligatoire du processus de traduction pris dans le sens large, à savoir de la commande à la livraison: «Le PST [prestataire de services de traduction] doit s'assurer que la traduction est révisée» (Comité européen de normalisation, 2006, p. 11).

Il est donc très probable, comme le pensent certains traductologues (Künzli, 2009; Mossop, 2007b) que, poussée par les nécessités de la norme européenne, la pratique révisante devienne de plus en plus fréquente: "The revision of the work of other translators may become increasingly important, at least in Europe, with the publication in 2006 of the new standard EN 15038 Translation services - Service requirements» (Mossop, 2007b, p. 6). Au Canada, où la révision est étudiée et enseignée depuis plus longtemps, la norme nationale pour les services de traduction CAN/CGSB-131.10-2008 porte également sur la révision.

Cependant, la question de la procédure de révision, c'est-àdire de la manière dont la révision doit être effectuée, reste posée. En effet, bien que dans un numéro spécial de JoSTrans consacré à la révision, Martin (2007, p. 58) estime que le principal atout de la norme est de mettre l'accent sur des définitions claires, Mossop, dans le même numéro, ne semble pas du même avis (2007b, p. 6). Nous partageons l'embarras de ce dernier: si l'on compare la définition du terme «réviser» dans la norme, à savoir « examiner une traduction pour vérifier son adéquation avec l'objet convenu, comparer le texte source (2.13) et le texte cible (2.14) et recommander des mesures correctives» (Comité européen de normalisation, 2006, p. 6), à la description qui en est donnée plus loin («Cette tâche peut comprendre, selon les nécessités du projet, 
la comparaison des textes source et cible [...]») (ibid., p. 11), l'on constate en effet que la comparaison des textes source et cible semble tantôt obligatoire, tantôt facultative.

Ces observations sont à la base d'une étude en cours sur les procédures de révision, dont les fondements ont été posés lors d'une étude exploratoire (Robert, 2008) et dont la principale question de recherche correspond en partie à la question que se posait Mossop, toujours dans ce numéro spécial de JoSTrans: «Is there a revising method that produces higher quality?» (2007b, p. 19). En d'autres termes, la procédure de révision a-t-elle un impact sur la révision, et, si oui, quelle procédure recommander?

Dans la présente contribution, nous nous intéresserons à la relecture unilingue, une procédure de révision qui consiste à ne relire que le texte cible, en faisant toutefois référence au texte source en cas de doute. Cette procédure, qui correspond à ce que propose la norme européenne lorsque le projet ne demande pas de comparaison des textes source et cible, est l'une des quatre procédures de révision les plus fréquentes ${ }^{2}$ sur le marché belge de la traduction (Robert, 2008), aux côtés de la relecture bilingue (comparaison des textes source et cible), de la relecture bilingue suivie d'une relecture unilingue et de la relecture bilingue précédée d'une relecture unilingue. La relecture unilingue est-elle efficace? Le cas échéant, dans quelles conditions et par rapport à quelle autre procédure? Telles seront les questions abordées dans le cadre de cet article.

Nous situerons d'abord la relecture unilingue dans la littérature sur la révision et en particulier sur les procédures de révision. Ensuite, nous formulerons des hypothèses relatives à cette procédure de révision tant en ce qui concerne le produit (c'est-àdire la qualité de la révision) que le processus de révision (c'està-dire, d'une part, la durée du processus de révision et, d'autre part, le potentiel de détection d'erreurs). La section suivante sera consacrée à la méthodologie utilisée dans l'étude. Elle sera suivie des résultats et enfin des conclusions.

\section{Les procédures de révision en traductologie}

La littérature sur les procédures de révision se fonde généralement

2. À noter que cette tendance a été confirmée par deux enquêtes: au sein de la banque nationale d'Autriche (Haussteiner, 2009) et sur le marché français de la traduction (Hernández Morin, 2009). 
sur les manuels de révision de Horguelin et Brunette (1998), Horguelin et Pharand (2009) et de Mossop (2001; 2007a) à l'exception bien entendu des publications antérieures, peu nombreuses toutefois. Graham (1989) semble recommander une relecture unilingue en guise de révision. Cette relecture pourra toutefois être précédée d'une relecture bilingue si le traducteur est jeune et inexpérimenté, à moins que le texte soit «sans réelle conséquence» (p. 64). Sager, en revanche, semble privilégier une procédure à deux étapes, composée d'une relecture bilingue suivie d'une relecture unilingue:

The first process, and the one which is exclusive to translation, is a form of parallel reading of source and target text document in order to verify that the content of the source text is reflected in the target text according to the instructions derived from the job specifications. [...] Once the process of comparison has been completed, any further adjustment is virtually the same as for any other form of text production, in which the emphasis is on the target text. (Sager, 1994, p. 237-238)

Gile (1995) recommande une procédure de révision fondée sur son modèle séquentiel de la traduction et qui consiste, comme chez Graham (1989), en une relecture unilingue assortie éventuellement d'un retour sur le texte source:

Revision, that is, the inspection and correction of a translation by a reviser after the translator has completed the task, is a repeat of the reformulation loop on groups of Translation units [...]. The reviser starts the process with the target text, testing successive groups of Translation Units for editorial acceptability and fidelity, generally at the level of sentences and above. Ideally, he or she focuses on acceptability, on the assumption that the translator is conscientious enough to have checked thoroughly the fidelity of the text. However, when dealing with a translator previously unknown to the reviser or having the reputation of not being very competent, when the text itself is critical, when the editorial quality of the targetlanguage text is found to be poor, or when there seem to be inconsistencies in the translation, the reviser must also focus on fidelity. This may at times lead back to the comprehension loop, when it appears that the translator may have chosen an unsatisfactory Meaning Hypothesis. 
For each group of Translation Units, then, revision can be said in a way to follow a process going in the opposite direction to that followed by the translation process itself. (Gile, 1995, p. 111)

En revanche, Rochard (1995) prône la révision en deux étapes, constituée d'une relecture logique du texte d'arrivée (dans un souci de cohérence interne et par rapport aux informations et à la logique du texte de départ), et une relecture contrastive du texte d'arrivée servant à vérifier qu'il n'y a rien dans la forme linguistique du texte de départ qui contredise la traduction.

Dans leur chapitre consacré aux principes généraux de la révision, et plus particulièrement aux "principes directeurs de la révision", Horguelin et Brunette (1998, p. 28) 3 abordent la question de la procédure de révision. La première étape consiste en une mise en situation qui correspond à la prise de connaissance des instructions de traduction et/ou de révision. Vient ensuite la révision proprement dite qui, idéalement, devrait se faire en plusieurs étapes: «1) lecture complète du texte original, 2) lecture comparative des deux textes (révision bilingue), 3) correction, 4) lecture du texte révisé» (ibid.,p. 39). Selon notre étude exploratoire, cette procédure ne semble pas appliquée sur le marché de la traduction. Les auteurs expliquent qu'en pratique, «on ne procède généralement qu'à une seule lecture (en principe comparative s'il s'agit d'une traduction) au cours de laquelle se fait la correction" (ibid., p. 39). La révision de la traduction peut également se faire à partir du texte traduit avec un retour sur l'original en cas de doute de la part de la personne qui révise. Les relectures unilingues et bilingues sont donc les plus fréquentes selon les auteurs du manuel. À noter toutefois que les auteurs prônent la relecture bilingue, car c'est la seule qui permette de s'assurer de la «fidélité » à l'original:

La tâche du réviseur est de s'assurer que les textes traduits qu'il révise ont les qualités d'une traduction professionnelle. Au premier rang de ces qualités vient la fidélité à l'original (fidélité n'étant pas synonyme de servilité). Il s'ensuit que la révision bilingue doit être comparative, car il n'y a pas d'autre moyen de vérifier que le transfert

3. Dans la $4^{\mathrm{e}}$ édition revue et augmentée de Pratique de la révision de Horguelin et Pharand (2009), ce passage consacré à la procédure de révision n'a pas changé sur le fond. Nous avons donc choisi de conserver la référence à l'édition précédente dans le corps du texte. 
a été correctement effectué, c'est-à-dire que la traduction transmet intégralement et exactement le message. On ne peut envisager à la rigueur qu'une exception à ce principe (outre le cas de la révision unilingue): la révision «linguistique» d'un texte qui a déjà fait l'objet d'une révision comparative par un expert n'ayant assuré que la fidélité au sens. (ibid., p. 40)

On ne s'étonnera pas dès lors que, dans sa classification des démarches évaluatives en traduction, Brunette (2000) fasse de la comparaison des textes source et cible une des caractéristiques de la révision proprement dite, qu'elle soit pragmatique ou didactique.

En 2001 comme en 2007, Mossop propose également une procédure de révision «standard», qu'il décrit comme idéale mais longue, et qui pourrait s'appliquer à la révision de textes non éphémères ou impliquant des décisions importantes. Cette procédure idéale (Mossop, 2007a, p. 159) commence par une relecture unilingue de la traduction, axée sur le paramètre de «logique» du groupe «contenu» et sur tous les paramètres du groupe «langue et style», à l'exception de celui qui concerne entre autres la grammaire et la ponctuation (mechanics). Cette relecture unilingue est suivie d'une relecture comparative durant laquelle le réviseur se concentrera sur les paramètres du groupe «transfert». Ensuite, le réviseur relira le texte cible dans sa totalité, en s'attelant au paramètre "mechanics» omis à l'étape précédente, ainsi qu'à la mise en pages, à la cohérence terminologique et à toute faute de langue introduite aux étapes précédentes. Il contrôlera les chiffres séparément. Il terminera par un contrôle de l'organisation du texte, suivi du contrôle automatique d'orthographe et de grammaire proposé par le traitement de texte et prendra soin de bien sauvegarder ses changements. Conscient qu'il s'agit là d'une procédure idéale, Mossop propose une série de questions que le réviseur pourra se poser pour choisir la procédure de révision appropriée. À noter qu'il reconnaît fonder ses conseils sur la logique, faute de recherches empiriques en la matière. En bref, il recommande les relectures doubles (bilingue + unilingue ou l'inverse) plutôt que les relectures simples (relecture unilingue seule ou relecture bilingue seule). Il ne compare pas véritablement les procédures simples, mais il explique qu'une relecture unilingue peut s'avérer très efficace: 
Unilingual re-reading (i.e. not looking at the source text unless a passage seems suspicious) can be very effective. At workshops, participants who have never tried it are often surprised at how many errors can be detected in this way. In particular, one can get quite good not only at spotting probable mistranslations in the draft but also at avoiding the introduction of mistranslations while correcting. (ibid., p. 146)

Ce point de vue ne semblait pas être partagé par Horguelin et Brunette (1998), comme nous l'avons déjà vu, ni par les auteurs de l'édition suivante (Horguelin et Pharand, 2009). Ce n'est pas non plus l'avis du groupe de recherche en révision humaine GREVIS. Fondé en 2005 et composé de Brunette, Gagnon et Hine, ce groupe de recherche avait pour but de mettre au point une méthode de révision accélérée permettant d'améliorer la qualité de l'opération. Le groupe avait formulé l'hypothèse selon laquelle une révision unilingue effectuée par une personne expérimentée et connaissant bien le sujet pouvait suffire et que le réviseur pouvait donc se passer de relecture comparative. Toutefois, très vite, les auteurs de l'étude se sont rendu compte que la relecture unilingue n'assurait pas le degré de qualité attendu, bien au contraire, et que la relecture bilingue lui était préférable:

Of the two types of revision examined in this study, bilingual revision yielded results that were more attractive. This was also shown to be the only type of second look that seemed profitable and indispensable for translation. Contrary to our expectations but according to the evidence, monolingual revision proved to be an irrational practice, even less helpful than no revision. It appeared that revisers accustomed to comparing translated and source texts lost their linguistic judgment when facing a "free-standing" text and corrupted the translation separated from its source text. (Brunette et al., 2005, p. 30)

Il s'agit là, à notre connaissance, de la première étude empirique sur le thème de la procédure de révision. La seconde étude empirique sur la révision a été menée par Künzli. En 2006, il rapporte déjà quelques observations relatives à la procédure de révision:

The following excerpt illustrates another issue: the question of the order in which revision procedures are carried out. There are some indications suggesting that 
from a quality point of view, it might be an advantage to start by reading the draft translation without looking at the source text: [...] If revisers start by reading the draft translation without looking at the source text, they have the unique opportunity to avoid coming under the spell of source-language structures. (2006b, p. 12-13)

Künzli abordera de nouveau la procédure de révision dans un article consacré à une étude empirique sur la qualité de la révision (2009), dans lequel il tente de répondre notamment à la question de recherche suivante: dans quel ordre les textes source et cible sont-ils lus? Y a-t-il un lien entre les procédures et la qualité de la révision? Künzli constate que le fait que le traducteur lise d'abord le texte source, d'abord le texte cible, ou lise les deux en parallèle, ne semble pas avoir d'effet sur la qualité. Ainsi, une différence de qualité significative n'a été observée que pour un seul texte (sur trois au total pour l'expérience): les réviseurs qui avaient commencé la révision par la lecture du texte source avaient obtenu des résultats significativement inférieurs aux autres réviseurs.

Pour clore cette partie sur les procédures de révision, nous terminerons par la proposition méthodologique de révision de traduction de Parra Galiano (2005, 2006, 2007a, 2007b) qui intègre, selon les aspects du texte cible à réviser, les paramètres et la procédure de révision à utiliser, ainsi que le profil de réviseur le plus approprié pour remplir la tâche de révision. Cette proposition méthodologique est le résultat d'une analyse descriptive, comparative et critique de la révision de traductions dans la traductologie, d'une part, et de l'évaluation des résultats de trois études de cas, d'autre part. Contrairement aux auteurs qui se sont penchés sur la révision et qui s'intéressent aux paramètres avant de s'intéresser à la procédure, Parra Galiano (2007b) dit faire l'inverse. Sa proposition méthodologique, composée de sept étapes, semble recommander la relecture unilingue, en tout cas dans un premier temps: 1) prendre connaissance du mandat de traduction, 2) lire le texte cible comme s'il s'agissait d'un texte original, 3) étudier la rentabilité de la révision, c'est-à-dire déterminer si oui ou non, sur la base des erreurs détectées à la première lecture, la traduction vaut la peine d'être révisée, 4) dans le cas d'une révision rentable, déterminer le degré de révision et les paramètres à utiliser, 5) réduire au minimum les modifications du texte cible, 6) être capable de justifier ses interventions et donc, 
ne rien modifier s'il n'y a pas d'argument de poids et 7) assumer sa responsabilité de réviseur.

En conclusion, on constate que les relectures doubles semblent recommandées, mais que faute de temps, ce sont les relectures simples qui l'emportent. Toutefois, les avis sont partagés quant à ces dernières, la relecture unilingue étant considérée tantôt comme efficace, tantôt vivement déconseillée.

\section{Hypothèses}

Comme nous l'écrivions dans l'introduction, l'objectif de cette étude est de déterminer l'impact de la procédure de révision la variable indépendante -, sur les variables dépendantes que sont le produit de révision (c'est-à-dire la qualité de la révision) et le processus de révision (c'est-à-dire, d'une part, la durée du processus de révision et, d'autre part, le potentiel de détection d'erreurs). Notre hypothèse principale, à savoir un effet significatif de la procédure de révision sur le produit et le processus de révision, a été confirmée (Robert, 2010). En ce qui concerne la qualité de la révision, la durée du processus de révision et le potentiel de détection d'erreurs ${ }^{4}$, nous avons constaté un effet significatif de la procédure de révision (test non paramétrique d'analyse de variance ANOVA Friedman significatif, $p<0,05$ ). Par conséquent, nous avons formulé plusieurs hypothèses quant à la relecture unilingue, non seulement en ce qui concerne une révision complète, mais aussi en ce qui concerne trois types de révisions dites partielles. Ainsi, nous avons déterminé au total quatre degrés de révision: la révision dite complète pour laquelle on tient compte de tous les paramètres de révision ${ }^{5}$ (exactitude, code linguistique - au sens strict comme au sens large - et adaptation fonctionnelle), la révision dite loyale pour laquelle on ne tient compte que du paramètre d'exactitude, la révision dite fonctionnelle pour laquelle on tient compte des paramètres de code linguistique et d'adaptation fonctionnelle et enfin, la révision dite minimale pour laquelle on ne tient compte que d'une partie des

4. Le potentiel de détection d'erreurs correspond à la capacité à détecter une erreur, sans que celle-ci ait pour autant été corrigée comme il se doit dans la version définitive.

5. Ces paramètres de révision sont basés sur les travaux de Horguelin et Brunette (1998), Horguelin et Pharand (2009), Brunette (2000) et du GREVIS (Brunette et al., 2005). 
paramètres d'exactitude et de code linguistique). ${ }^{6}$ Ces hypothèses sont les suivantes:

1) En ce qui concerne la qualité de la révision, la relecture unilingue est significativement moins efficace que la relecture bilingue en révision complète, en révision loyale et en révision minimale; en revanche, elle est significativement plus efficace que la relecture bilingue en révision fonctionnelle. En effet, même si ces deux procédures comptent le même nombre de lectures du texte cible, la relecture unilingue est moins efficace, en révision complète, que la relecture bilingue, car elle ne permet sans doute pas de détecter les erreurs liées au paramètre d'exactitude. C'est en tout cas l'avis de Horguelin et Brunette (1998) et de Horguelin et Pharand (2009) mais pas de Mossop (2007a). Même si la relecture unilingue permet au réviseur de se concentrer sur les paramètres de code linguistique et d'adaptation fonctionnelle, on peut s'attendre à ce que ce gain vis-à-vis de la relecture bilingue ne soit pas suffisant pour compenser le risque sur le plan de l'exactitude. En révision loyale et en révision minimale, l'argument est le même. En revanche, en révision fonctionnelle, la relecture unilingue est plus efficace que la relecture bilingue, car elle permet au réviseur de se concentrer uniquement sur les paramètres de code linguistique et d'adaptation fonctionnelle, alors que la relecture bilingue demande un effort cognitif supplémentaire, celui de la comparaison des textes source et cible. Par ailleurs, en appliquant la relecture unilingue, le réviseur n'est pas influencé par le texte source et, donc, les caractéristiques de la langue source. Le risque d'interférences est par conséquent moins grand. Enfin, la relecture

6. Le terme loyal a été choisi en raison de la théorie de la traduction constituant le cadre théorique général de l'étude, soit la théorie fonctionnaliste telle que définie par Nord (2005). Nord souligne que la «loyauté» porte sur la responsabilité du traducteur envers ses partenaires de l'interaction traductionnelle et qu'il ne faut pas la confondre avec la «fidélité», qui fait référence à la relation entre texte source et texte cible. Toutefois, nous avons choisi de qualifier une traduction de loyale lorsquelle répondait au mandat de traduction en termes d'exactitude et de contenu. De même, nous avons choisi de parler d'une traduction fonctionnelle lorsqu'elle répondait au mandat de traduction en termes de code linguistique et d'adaptation fonctionnelle (traduction qui tient compte du destinataire et de l'usage que celui-ci en fera). Bien que cette appellation puisse paraître quelque peu abusive, elle nous permettait de refléter les deux grands concepts de Nord, que sont la loyauté et la fonction. 
unilingue est moins efficace que les procédures doubles, quel que soit le niveau de révision envisagé, puisqu'elle ne comporte qu'une seule relecture, au lieu de deux.

2) En ce qui concerne le potentiel de détection d'erreurs, nous avons formulé les mêmes hypothèses que pour la qualité de la révision.

3) En ce qui concerne la durée du processus de révision, la relecture unilingue est significativement plus efficace (donc prend moins de temps) que la relecture bilingue, car le réviseur ne doit pas faire de va-et-vient entre les deux textes et se concentre surtout sur les paramètres de code linguistique et d'adaptation fonctionnelle. Par ailleurs, la relecture unilingue est significativement plus efficace que les relectures doubles puisqu'elle ne compte qu'une seule relecture.

\section{Méthodologie}

D'un point de vue méthodologique, et en particulier en ce qui concerne la stratégie générale de vérification de nos hypothèses, nous avons opté pour la stratégie quasi expérimentale, les expériences conduites par nos soins consistant à observer le produit et le processus de révision (deux variables dépendantes) lorsque l'on fait varier la procédure de révision (la variable indépendante). Les instruments de collecte de données sont au nombre de quatre, offrant ainsi la possibilité de ce que Alves (2003) notamment a appelé la triangulation: l'analyse de la révision en tant que produit est fondée sur les changements apportés dans la version finale, tels que révélés par la fonction de comparaison de documents du logiciel de traitement de texte MS Word, tandis que l'analyse de la révision en tant que processus est basée sur des protocoles de réflexion à voix haute (Think Aloud Protocols) et sur les données générées par le logiciel de saisie de frappe Inputlog (Leijten et Van Waes, 2006). Enfin, des données complémentaires sur les sujets ont été collectées par le biais d'entretiens menés en fin d'expérience.

L'expérimentation consistait à faire réviser quatre textes cibles par seize réviseurs professionnels, chaque fois selon une des quatre procédures les plus courantes sur le marché belge (v. Introduction): la relecture unilingue (nommée procédure $\mathrm{A}$ ), la relecture bilingue (procédure $\mathrm{B}$ ), la relecture bilingue suivie d'une relecture unilingue (procédure $\mathrm{C}$ ) et la relecture bilingue précédée d'une relecture 
unilingue (procédure D). Les quatre textes cibles devaient être comparables: il s'agissait de quatre communiqués de presse approximativement de même longueur, traduits en français en 2008 par des étudiants francophones de Master en traduction. Ces quatre textes appartenaient donc au même genre et poursuivaient la même fonction, identique à celle des textes sources ${ }^{7}$. Par ailleurs, ils contenaient approximativement la même proportion d'erreurs authentiques (dénommées «items» dans l'étude, cf. infra) et le plus objectives possible, car ayant fait l'objet d'un consensus au sein d'un groupe de professionnels de la traduction et/ou de l'enseignement du français en milieu universitaire. Ces erreurs ou items ont été classés en fonction des trois paramètres de révision décrits précédemment: exactitude, code linguistique et adaptation fonctionnelle.

\subsection{Participants}

Lobjectif était de recruter seize réviseurs présentant un profil plus ou moins similaire : réviseur professionnel francophone, expérience de un an au moins en révision, habitude de révision du néerlandais au français. Une interview rétrospective nous a permis de dresser le profil des sujets, selon les critères suivants: le statut du réviseur (employé ou indépendant), l'âge, le sexe, le type d'études, le nombre d'années d'expérience en traduction et en révision, le statut du néerlandais (langue source) dans leur formation principale, le

7. Les textes sources étaient quatre communiqués de presse de même longueur (approximativement 500 mots) rédigés en néerlandais en 2000 par la même agence de communication belge. En tant que communiqués de presse, les textes sources appartiennent au même genre et poursuivent la même fonction, à savoir, informer. Une analyse des facteurs intratextuels et extratextuels réalisée selon le modèle de Nord (2005) a confirmé le caractère comparable de nos textes sources à de nombreux points de vue. Par ailleurs, en se fondant sur la méthode d'évaluation de la difficulté d'une tâche de traduction de Nord, on constate qu'ils sont également comparables $\mathrm{du}$ point de vue des problèmes de traduction, qu'ils soient pragmatiques, liés aux conventions, linguistiques ou spécifiques au texte. Enfin, s'il faut en croire la formule de lisibilité de Douma-Flesch (Defrancq et Van Laecke, 2009, p. 36) appliquée à la langue néerlandaise, ces textes sources sont très comparables: ils sont tous les quatre classés dans la catégorie « difficile» avec un score se situant entre 30 et 45 (respectivement 36, 45; 41, 99; 38, 27; 44, 06), ce qui veut dire qu'ils doivent pouvoir être lus et compris par un lecteur ayant suivi un enseignement supérieur non universitaire. 
statut du français (langue maternelle pour tous les sujets) et enfin leurs habitudes en matière de procédures de révision.

\subsection{Déroulement des expériences et matériel}

Les expériences ont eu lieu entre janvier et juin 2009, à la meilleure convenance du réviseur. Chaque expérience a donc été planifiée par le réviseur comme n'importe quelle autre tâche de révision commandée par un client «ordinaire ${ }^{8}$. Il ne s'agissait donc pas d'une commande «express » non prévue au planning. Par ailleurs, aucune limite de temps nétait imposée aux réviseurs, mais nous leur avions quand même donné une indication sur la durée de l'expérience (pour la révision des quatre textes: environ deux heures trente). Les quatre textes cibles ont été révisés un à un, avec chaque fois une courte pause de cinq minutes nous permettant d'effectuer les opérations nécessaires à la collecte des données processus (notamment la sauvegarde de fichiers).

La plupart des expériences ont eu lieu sur le lieu de travail habituel du réviseur, de façon à ce que l'expérience soit aussi proche que possible de la réalité. Avant le début de l'expérience, le réviseur recevait, en version papier, quatre documents qu'il avait déjà reçus auparavant par voie électronique lors du recrutement: un formulaire de consentement, une brève explication sur la méthode de réflexion à voix haute, le mandat de révision (mentionnant les paramètres de révision à appliquer, à savoir exactitude, code linguistique - lisibilité compris - et adaptation fonctionnelle) et un document expliquant brièvement le déroulement de l'expérience. En outre, il recevait une fiche de travail personnalisée lui indiquant quel texte réviser avec quelle procédure et dans quel ordre. Toutes les instructions étaient également fournies oralement avant chaque expérience. Les textes sources étaient disponibles sur papier, mais les textes cibles ne l'étaient que sous format électronique, dans un fichier MS Word. La décision de ne pas autoriser les réviseurs à travailler sur papier avait été prise sur la base de notre étude exploratoire (Robert, 2008) qui avait révélé, par le biais d'une enquête parmi des agences de traduction belges, que la révision sur papier était de plus en plus rare.

Les réviseurs pouvaient utiliser tous les outils traditionnels habituels, mais devaient impérativement travailler sur notre

8. Une rémunération, basée sur les tarifs en vigueur sur le marché, était donc prévue. 
ordinateur portable (sur lequel était installé le logiciel Inputlog, cf. supra). Toutefois, ils avaient accès à tous les dictionnaires électroniques habituels, à savoir Le Petit Robert électronique, les Van Dale néerlandais-français et français-néerlandais ainsi que le Van Dale explicatif (il s'agit des dictionnaires généraux de référence en traduction néerlandais-français et français-néerlandais), et bien sûr, ils pouvaient consulter l'internet.

Pour l'enregistrement des verbalisations, nous avons utilisé un dictaphone numérique. Les réviseurs portaient un casque avec microphone pour que même des verbalisations murmurées restent audibles. Les réviseurs appuyaient eux-mêmes sur le bouton d'enregistrement «rec» avant de démarrer l'expérience et sur le bouton «stop» après chaque expérience. En effet, nous n'étions jamais présente dans la même pièce que le réviseur, pour qu'il se sente libre de penser tout haut. Pour l'enregistrement des frappes clavier et des mouvements de souris, nous avons utilisé le logiciel de saisie de frappes et de mouvement de souris Inputlog (Leijten \& Van Waes, 2006; voir aussi www.inputlog.net), qui a l'avantage de ne pas interférer dans le processus de révision, puisqu'il fonctionne dans MS Word sans que le réviseur ne le voie.

\subsection{Méthode d'analyse}

Pour les données produit, nous avons comparé la moyenne des scores de chaque réviseur en fonction de la procédure utilisée. Pour calculer le score de chaque réviseur, nous avons additionné les «révisions pertinentes»: il s'agit de toute intervention qui consiste à corriger une erreur (ou item ${ }^{9}$ ) de manière pertinente. Ainsi, un réviseur effectuant 10 révisions pertinentes dans un texte contenant 20 items et obtenait un score de $50 \%$. Pour les données processus, en particulier en ce qui concerne la durée de la révision, nous avons comparé la moyenne des durées de révision de chaque réviseur en fonction de la procédure utilisée. En ce qui concerne le potentiel de détection de chaque procédure (c'est-à-dire la capacité à détecter une erreur, sans pour autant $\mathrm{y}$ apporter une solution), nous avons également comparé la moyenne des scores de chaque

9. Plutôt que de prévoir une évaluation de la qualité a posteriori, nous avons opté pour la méthode des items: il s'agit d'erreurs authentiques sélectionnées en fonction du paramètre de révision auquel elles correspondent et pour lesquelles un consensus a été trouvé au sein d'un panel de neuf spécialistes. V. Segers (2007). 
réviseur en fonction de la procédure utilisée. Pour calculer ce score, nous avons additionné les révisions pertinentes, les «sousrévisions» (toute intervention qui consiste à corriger une erreur (item) de manière non pertinente) et les simples "détections" (items pour lesquels une détection a été constatée sur la base des verbalisations ou de l'enregistrement des saisies de frappe). Ainsi, un réviseur effectuant 10 révisions pertinentes, 3 sous-révisions et 2 détections dans un texte contenant 20 items obtenait un score de $75 \%$. La typologie des interventions est inspirée des travaux de Horguelin et Brunette (1998) ou encore Künzli (2006a) qui distinguent d'autres types d'interventions ${ }^{10}$ que nous n'aborderons pas dans le cadre de cet article.

\section{Résultats}

\subsection{Hypothèses relatives à la qualité de la révision}

Pour rappel, nous avons posé l'hypothèse selon laquelle la relecture unilingue est significativement moins efficace que la relecture bilingue en révision complète, en révision loyale et en révision minimale, mais qu'elle est significativement plus efficace que la relecture bilingue en révision fonctionnelle. En outre, la relecture unilingue est significativement moins efficace que les procédures doubles, quel que soit le niveau de révision envisagé. Les résultats sont repris dans la Figure 1 ci-dessous $(\mathrm{PA}=$ procédure $\mathrm{A}=$ relecture unilingue; $\mathrm{PB}=$ procédure $\mathrm{B}=$ relecture bilingue; $\mathrm{PC}=$ procédure $\mathrm{C}=$ relecture bilingue suivie d'une relecture unilingue; $\mathrm{PD}=$ procédure $\mathrm{D}$ = relecture bilingue précédée d'une relecture unilingue.

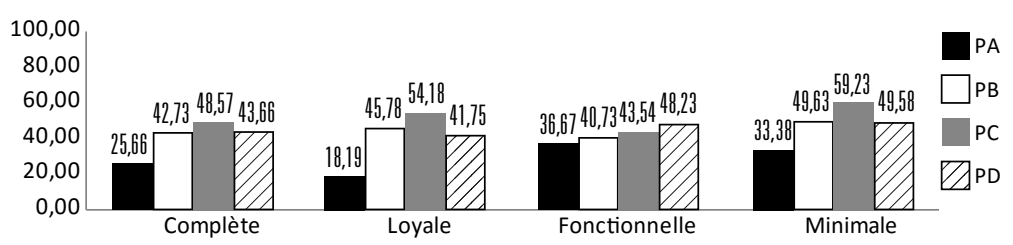

Figure 1. Comparaison des scores moyens (\%) de chaque procédure en ce qui concerne la qualité du produit de révision

10. Il s'agit des «hyperrévisions », toute intervention qui n'améliore pas mais ne détériore pas non plus le texte; et des «surrévisions», toute introduction d'erreurs. 
Comme nous l'écrivions précédemment (cf. supra), le test non paramétrique ANOVA Friedman pour mesures répétées s'est révélé significatif $(p<0,05)$, excepté toutefois dans le cas de la révision fonctionnelle, comme l'illustre le Tableau 1.

Tableau 1. Résultats du test non paramétrique ANOVA Friedman, par degré de révision (données "produit»)

\begin{tabular}{llllll}
\hline Révision & Chi-carré & $\begin{array}{l}\text { Degré de Population } \\
\text { liberté } \\
(d l)\end{array}$ & $\begin{array}{l}\text { Valeur de la } \\
\text { probabilité }\end{array}$ & Significatif \\
& $\left(\chi^{2}\right)$ & $(p)$ & 0,004 & $*$ \\
\hline Complète & 12,47 & 3 & 16 & $* 003$ & $*$ \\
Loyale & 13,13 & 3 & 16 & 0,003 \\
Fonctionnelle & 2,98 & 3 & 16 & 0,402 & $*$ \\
Minimale & 8,05 & 3 & 16 & 0,042 & $*$ \\
\hline
\end{tabular}

Le type de procédure a donc un effet sur le produit ou la qualité de la révision, excepté dans le cas d'une révision fonctionnelle. Afin d'identifier des différences significatives entre deux moyennes déterminées et donc entre la relecture unilingue et les autres procédures, nous avons effectué le test paramétrique d'analyse de la variance ANOVA pour un facteur pour mesures répétées (oneway ANOVA for repeated measures) qui offre la possibilité de tests additionnels (post hoc) comparant les moyennes deux à deux. Ce test était également significatif, excepté dans le cas de la révision fonctionnelle, comme l'illustre le Tableau 2. Les résultats des tests additionnels sont repris dans le Tableau 3.

Tableau 2. Résultats du test paramétrique ANOVA, par degré de révision (données "produit»)

\begin{tabular}{llllll}
\hline Révision & Chi-carré & $\begin{array}{l}\text { Degré de } \\
\text { liberté } \\
(d l)\end{array}$ & $\begin{array}{l}\text { Population } \\
(N)\end{array}$ & $\begin{array}{l}\text { Valeur de la } \\
\text { probabilité }\end{array}$ & Significatif \\
$(p)$ & $*$ \\
\hline Complète & 5,46 & 3 & 16 & 0,003 & $*$ \\
Loyale & 6,79 & 3 & 16 & 0,001 & $*$ \\
Fonctionnelle & 1,12 & 3 & 16 & 0,353 & $*$ \\
Minimale & 3,03 & 3 & 16 & 0,039 & $*$ \\
\hline
\end{tabular}


Tableau 3. Résultats des tests post hoc de Sidak ${ }^{11}$

\begin{tabular}{llllll}
\hline Révision & $\begin{array}{l}\text { Comparaison } \\
\text { des procédures }\end{array}$ & $\begin{array}{l}\text { Différence } \\
\text { moyenne }\end{array}$ & $\begin{array}{l}\text { Erreur } \\
\text { standard }\end{array}$ & $\begin{array}{l}\text { Valeur de la } \\
\text { probabilité } \\
\text { (Sidak) } \\
p\end{array}$ & \\
\hline Complète & A-B & $-17,064$ & 5,974 & 0,070 & \\
& A-C & $-22,908$ & 5,902 & 0,009 & $*$ \\
& A-D & $-17,992$ & 4,454 & 0,006 & $*$ \\
\hline Loyale & A-B & $-27,585$ & 7,974 & 0,021 & $*$ \\
& A-C & $-35,985$ & 7,034 & 0,001 & $*$ \\
& A-D & $-23,551$ & 5,991 & 0,008 & $*$ \\
\hline Fonctionnelleatif & A-B & $-4,062$ & 7,390 & 0,995 & \\
& A-C & $-6,875$ & 8,101 & 0,958 & \\
& A-D & $-11,563$ & 5,637 & 0,302 & \\
\hline Minimale & A-B & $-16,245$ & 9,291 & 0,471 & \\
& A-C & $-25,843$ & 8,596 & 0,052 & $\left(^{*}\right)$ \\
& A-D & $-16,195$ & 6,376 & 0,128 & \\
\hline
\end{tabular}

Contrairement aux hypothèses de départ et à ce que la Figure 1 semblait indiquer, on constate que la relecture unilingue (A) n'est pas significativement moins efficace que la relecture bilingue (B) en révision complète et en révision minimale. On observe également qu'il n'y a pas de différence significative entre ces deux procédures en révision fonctionnelle. En revanche, conformément à notre hypothèse de départ, la relecture unilingue est significativement moins efficace que la relecture bilingue en révision loyale. Par rapport aux procédures doubles $(\mathrm{C}$ et $\mathrm{D})$, la relecture unilingue est significativement moins efficace en révision complète et loyale, conformément aux hypothèses, mais elle n'est pas significativement moins efficace en révision fonctionnelle et minimale. Toutefois, comparée à la procédure $\mathrm{C}$, la relecture unilingue est probablement moins efficace en révision minimale, la valeur de la probabilité étant très proche du seuil de signification.

11. Le test effectué sous SPSS 15.0 autorise le choix entre trois niveaux d'ajustement de l'intervalle de confiance: Tukey LSD (pas d'ajustement), Bonferroni ou Sidak. Ce dernier est recommandé lorsque le chercheur craint une perte de puissance associée à Bonferroni. V. Field (2009, p. 473). 


\subsection{Hypothèses relatives au potentiel de détection d'erreurs}

En ce qui concerne le potentiel de détection d'erreurs, nous avons formulé les mêmes hypothèses que pour la qualité de la révision. Les résultats sont repris dans la Figure 2 ci-dessous:

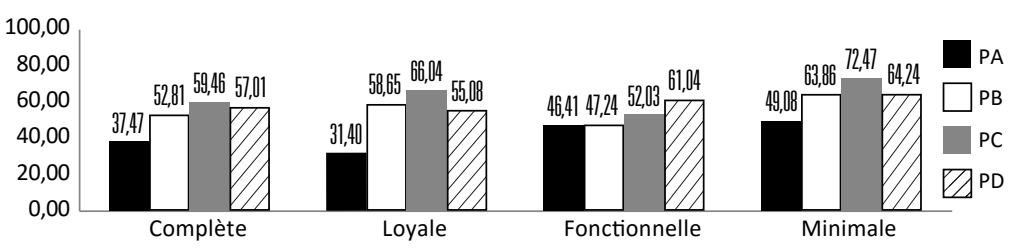

Figure 2. Comparaison des scores moyens (\%) de chaque procédure en ce qui concerne le potentiel de détection d'erreurs

Le test non paramétrique ANOVA Friedman pour mesures répétées s'est révélé significatif $(p<0,05)$ dans le cas d'une révision complète et loyale, mais pas dans le cas des révisions fonctionnelle et minimale, comme l'illustre le Tableau 4.

Tableau 4. Résultats du test non paramétrique ANOVA Friedman, par degré de révision (données "potentiel de détection »)

\begin{tabular}{llllll}
\hline Révision & Chi-carré & $\begin{array}{l}\text { Degré de } \\
\text { liberté } \\
(d l)\end{array}$ & $\begin{array}{l}\text { Population } \\
\left(\chi^{2}\right)\end{array}$ & $\begin{array}{l}\text { Valeur de la } \\
\text { probabilité }\end{array}$ & Significatif \\
$(p)$ & $*$ \\
Complète & 10,13 & 3 & 16 & 0,015 & $*$ \\
Loyale & 11,78 & 3 & 16 & 0,007 & $*$ \\
Fonctionnelle & 6,63 & 3 & 16 & 0,080 & \\
Minimale & 6,87 & 3 & 16 & 0,070 & \\
\hline
\end{tabular}

L'hypothèse est donc confirmée dans deux cas de figure sur quatre. Les résultats du test paramétrique d'analyse de la variance ANOVA pour un facteur pour mesures répétées (one-way ANOVA for repeated measures) sont repris dans le Tableau 5. Les résultats des tests additionnels comparant les moyennes deux à deux sont repris au Tableau 6. 
Tableau 5. Résultats du test paramétrique ANOVA, par degré de révision (données "potentiel de détection")

\begin{tabular}{llllll}
\hline Révision & Chi-carré & $\begin{array}{l}\text { Degré de } \\
\text { liberté } \\
(d l)\end{array}$ & $\begin{array}{l}\text { Population } \\
(N)\end{array}$ & $\begin{array}{l}\text { Valeur de la } \\
\text { probabilité } \\
(p)\end{array}$ & Significatif \\
\hline Complète & 5,81 & 3 & 16 & 0,002 & $*$ \\
Loyale & 6,19 & 3 & 16 & 0,001 & $*$ \\
Fonctionnelle & 2,31 & 3 & 16 & 0,089 & \\
Minimale & 2,68 & 3 & 16 & 0,058 & \\
\hline
\end{tabular}

Tableau 6. Résultats des tests post hoc de Sidak

\begin{tabular}{llllll}
\hline Révision & $\begin{array}{l}\text { Comparaison } \\
\text { des procédures }\end{array}$ & $\begin{array}{l}\text { Différence } \\
\text { moyenne }\end{array}$ & $\begin{array}{l}\text { Erreur } \\
\text { standard }\end{array}$ & $\begin{array}{l}\text { Valeur de la } \\
\text { probabilité } \\
\text { (Sidak) } \\
(p)\end{array}$ & Significatif \\
\hline Complète & A-B & $-15,340$ & 6,141 & 0,139 & \\
& A-C & $-21,987$ & 6,817 & 0,034 & $*$ \\
& A-D & $-19,535$ & 3,644 & 0,000 & $*$ \\
\hline Loyale & A-B & $-27,247$ & 8,464 & 0,034 & $*$ \\
& A-C & $-34,643$ & 9,838 & 0,018 & $*$ \\
& A-D & $-23,681$ & 5,228 & 0,002 & $*$ \\
\hline Fonctionnelle & A-B & $-0,833$ & 7,030 & 1,000 & \\
& A-C & $-5,625$ & 8,021 & 0,983 & \\
& A-D & $-14,635$ & 5,674 & 0,119 & \\
\hline Minimale & A-B & $-14,782$ & 8,511 & 0,479 & \\
& A-C & $-23,388$ & 9,778 & 0,169 & \\
& A-D & $-15,154$ & 6,943 & 0,243 & \\
\hline
\end{tabular}

Contrairement aux hypothèses de départ et à ce que la Figure 1 semblait indiquer, on constate que la relecture unilingue (A) n'est pas significativement moins efficace que la relecture bilingue (B) en révision complète et en révision minimale. On observe également qu'il n'y a pas de différence significative entre ces deux procédures en révision fonctionnelle. En revanche, conformément à notre hypothèse de départ, la relecture unilingue 
est significativement moins efficace que la relecture bilingue en révision loyale. Par rapport aux procédures doubles (C et $\mathrm{D})$, la relecture unilingue est significativement moins efficace en révision complète et loyale, conformément aux hypothèses, mais elle n'est pas significativement moins efficace en révision fonctionnelle et minimale. Par conséquent, ces résultats sont comparables à ce qui a été observé pour la qualité de la révision.

\subsection{Hypothèses relatives à la durée du processus}

Pour rappel, en ce qui concerne la durée du processus de révision, nous avons posé l'hypothèse que la relecture unilingue est significativement plus efficace (donc prend moins de temps) que la relecture bilingue et que les relectures doubles. Les résultats sont repris dans la Figure 3.

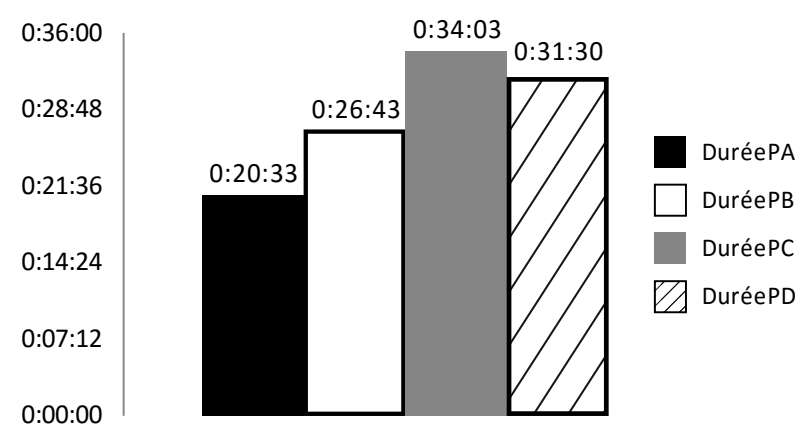

Figure 3. Durée moyenne de la révision, par procédure

Le test non paramétrique ANOVA Friedman est significatif $\left(\chi^{2}(3, N=16)=16,73 ; p<0,001\right)$, ce qui veut dire que la variable indépendante, à savoir le type de procédure, a un effet sur la variable dépendante, ici la durée du processus de révision. Il est pratiquement impossible de mesurer la durée du processus en ne tenant compte que de certains paramètres de révision, et donc en distinguant différents degrés de révision. Nous ne l'avons donc pas 
fait $^{12}$. Le test paramétrique d'analyse de variance ANOVA pour un facteur à mesures répétées s'est lui aussi révélé significatif: $F(3,16)=5,46 ; p=0,003$. Les tests post hoc de Sidak révèlent des écarts significatifs pour les paires A-C et A-D, comme l'illustre le Tableau 7 ci-dessous.

Tableau 7. Résultats des tests post hoc de Sidak

\begin{tabular}{lllll}
\hline $\begin{array}{l}\text { Comparaison } \\
\text { des procédures }\end{array}$ & $\begin{array}{l}\text { Différence } \\
\text { moyenne }\end{array}$ & Erreur standard & $\begin{array}{l}\text { Valeur de la } \\
\text { probabilité } \\
\text { (Sidak) } \\
(\mathrm{p})\end{array}$ & Significatif \\
\hline A-B & $-370,375$ & 215,622 & 0,491 & \\
A-C & $-810,625$ & 215,975 & 0,011 & $*$ \\
A-D & $-657,500$ & 184,607 & 0,017 & $*$ \\
\hline
\end{tabular}

Par conséquent, notre hypothèse n'est vérifiée qu'à moitié: la relecture unilingue ne prend pas significativement moins de temps que la relecture bilingue, mais elle prend significativement moins de temps que les procédures doubles.

\section{Conclusions}

Dans cette contribution, nous nous sommes intéressée à la relecture unilingue, une procédure de révision qui consiste à ne relire que le texte cible, en faisant toutefois référence au texte source en cas de doute. Cette procédure, qui fait partie des procédures de révision les plus utilisées sur le marché belge de la traduction, pourrait correspondre à la procédure prônée par la norme européenne EN 15038 sur les services de traduction, dans la mesure où cette dernière n'est pas claire quant à l'obligation de comparaison des textes source et cible. Les traductologues sont divisés à son égard: elle serait certes moins efficace que les procédures impliquant deux relectures, mais, par rapport à la relecture bilingue (relecture comparative unique), elle serait tantôt vivement déconseillée,

12. Nous avons demandé aux réviseurs de réviser les traductions en tenant compte de tous les paramètres de qualité mentionnés et nous avons mesuré la durée de chaque révision. Ce n'est qu'a posteriori que nous avons également évalué les révisions en ne tenant compte que de certains paramètres. Il n'est donc pas possible de ne mesurer la durée de la révision que pour certains paramètres. 
tantôt considérée comme efficace. Dans notre étude, la relecture unilingue a donc été comparée à la relecture bilingue ainsi qu'aux relectures doubles afin de déterminer si elle était plus efficace et, le cas échéant, dans quelles conditions et par rapport à quelle autre procédure. Les résultats de nos recherches sont repris dans le Tableau de synthèse (8) ci-dessous.

Tableau 8. Comparaison qualité-potentiel/durée

\begin{tabular}{lllllllll}
\hline $\begin{array}{l}\text { Révision } \\
\text { complète }\end{array}$ & \multicolumn{2}{l}{$\begin{array}{l}\text { Révision } \\
\text { loyale }\end{array}$} & & \multicolumn{2}{l}{$\begin{array}{l}\text { Révision } \\
\text { fonctionnelle }\end{array}$} & \multicolumn{2}{l}{$\begin{array}{l}\text { Révision } \\
\text { minimale }\end{array}$} \\
Qualité & Potentiel & Qualité & Potentiel & Qualité & Potentiel & Qualité & Potentiel & Durée \\
\hline $\mathrm{A}=\mathrm{B}$ & $\mathrm{A}=\mathrm{B}$ & $\mathrm{A}<\mathrm{B}$ & $\mathrm{A}<\mathrm{B}$ & $\mathrm{A}=\mathrm{B}$ & $\mathrm{A}=\mathrm{B}$ & $\mathrm{A}=\mathrm{B}$ & $\mathrm{A}=\mathrm{B}$ & $\mathrm{A}=\mathrm{B}$ \\
$\mathrm{A}<\mathrm{C}$ & $\mathrm{A}<\mathrm{C}$ & $\mathrm{A}<\mathrm{C}$ & $\mathrm{A}<\mathrm{C}$ & $\mathrm{A}=\mathrm{C}$ & $\mathrm{A}=\mathrm{C}$ & $\mathrm{A}<\mathrm{C}$ ? & $\mathrm{A}=\mathrm{C}$ & $\mathrm{A}>\mathrm{C}$ \\
$\mathrm{A}<\mathrm{D}$ & $\mathrm{A}<\mathrm{D}$ & $\mathrm{A}<\mathrm{D}$ & $\mathrm{A}<\mathrm{D}$ & $\mathrm{A}=\mathrm{D}$ & $\mathrm{A}=\mathrm{D}$ & $\mathrm{A}=\mathrm{D}$ & $\mathrm{A}=\mathrm{D}$ & $\mathrm{A}>\mathrm{D}$ \\
\hline
\end{tabular}

Note: Les signes < et > signifient respectivement «sont moins efficaces» et «plus efficaces»; par conséquent, en ce qui concerne la durée, le signe < veut dire «est moins efficace, car prend plus de temps» et le signe > veut dire «est plus efficace, car prend moins de temps».

En révision complète, s'il doit choisir entre la relecture unilingue (A) et la relecture bilingue (B), le réviseur devra peser le pour et le contre: la relecture unilingue semble à première vue moins efficace sur le plan de la qualité de la révision et du potentiel de détection d'erreurs, mais elle semble aussi prendre moins de temps. Toutefois, dans un cas comme dans l'autre, les différences ne sont pas significatives d'un point de vue statistique. Néanmoins, étant donné que le seuil de signification est proche en ce qui concerne la qualité de la révision $(p=0,07)$, nous conseillons quand même la relecture bilingue. $\mathrm{S}^{\prime}$ il doit choisir entre la relecture unilingue ou une relecture double $(\mathrm{C}$ ou $\mathrm{D})$, le réviseur doit savoir que la relecture unilingue est significativement moins efficace que les deux autres sur le plan de la qualité et du potentiel de détection d'erreurs, mais qu'elle prend aussi significativement moins de temps.

En révision loyale, la relecture unilingue semble la moins efficace. Si le réviseur doit choisir entre la relecture unilingue (A) et la relecture bilingue (B), nous lui conseillons de choisir la seconde: elle est significativement plus efficace sur le plan de la qualité et du potentiel de détection d'erreurs et ne prend pas significativement 
plus de temps. S'il doit choisir entre la relecture unilingue (A) et les relectures doubles $(\mathrm{C}$ ou $\mathrm{D})$, le réviseur doit savoir que la relecture unilingue est significativement moins efficace que les deux autres sur le plan de la qualité et du potentiel de détection d'erreurs, mais qu'elle prend aussi significativement moins de temps.

En révision fonctionnelle, nous avons constaté, en ce qui concerne la qualité, le potentiel de détection d'erreurs et la durée, qu'il n'y avait pas de différence significative entre la relecture unilingue $(\mathrm{A})$ et la relecture bilingue (B). On préférera donc la première. En revanche, s'il n'y a pas de différence significative entre la relecture unilingue et les relectures doubles en ce qui concerne la qualité et le potentiel de détection, il y en a une en ce qui concerne la durée: la relecture unilingue n'est pas significativement moins efficace que ces deux procédures et elle prend significativement moins de temps. Elle sera donc recommandée.

Enfin, en révision minimale, on arrive sensiblement aux mêmes conclusions qu'en révision fonctionnelle. La relecture unilingue, qui semble la moins efficace à première vue, ne l'est pas d'un point de vue statistique. Seul un écart pratiquement significatif $(p=0,052)$ a été constaté avec la procédure $C$ (relecture double composée d'une relecture bilingue suivie d'une relecture unilingue). Si le réviseur doit choisir entre la relecture unilingue et la relecture bilingue, il pourra utiliser la procédure de son choix, puisqu'aucune différence significative n'a été constatée, ni sur le plan de la qualité ou du potentiel, ni en termes de durée. Toutefois, il se souviendra que la relecture unilingue semble à première vue plus rapide que la relecture bilingue, mais semble également moins efficace. S'il doit choisir entre la relecture unilingue (A) et la procédure $\mathrm{C}$, le réviseur devra faire la part des choses : la première est peut-être moins efficace en termes de qualité (différence quasi significative), mais est significativement plus rapide. S'il a le choix entre la relecture unilingue $(A)$ et la procédure $D$, il optera pour la première, en raison du gain de temps significatif associé à la relecture unilingue.

En conclusion, si la relecture unilingue semble rapide et fonctionnelle, elle s'avère déloyale. Au réviseur de sélectionner la procédure appropriée en connaissance de cause, c'est-à-dire en considérant les avantages et les inconvénients de chaque procédure, compte tenu du temps qui lui est imparti. Toutefois, les résultats de cette première analyse sont fondés sur un test statistique 
(ANOVA) ne tenant pas compte de la hiérarchie des données: chaque score en effet est celui d'un réviseur en particulier ayant révisé un texte en particulier, avec la méthode imposée pour la tâche en question. En dehors de l'effet de la procédure utilisée, qui est l'objet de la présente étude, le réviseur et le texte révisé ont-ils eu un effet significatif? Pour répondre à ces questions, une seconde analyse statistique serait nécessaire, l'analyse «multiniveau». Cette analyse ouvre une nouvelle piste de recherche, mais n'entre pas dans le cadre de la présente contribution. Toutefois, elle fera sans aucun doute l'objet de publications ultérieures. ${ }^{13}$

\section{Références}

Alves, Fabio, dir. (2003). Triangulating Translation. Perspectives in Process Oriented Research. Amsterdam/Philadelphie, John Benjamins.

Brunette, Louise (1997). Contribution à la pédagogie de la révision anglais-français en pays bilingue: le cas du Canada. Villeneuve d'Arcq, Éditions du Septentrion.

Brunette, Louise (2000). "Towards a Terminology for Translation Quality Assessment». The Translator: Studies in Intercultural Communication, 6, 2, p. 169-182.

Brunette, Louise (2007). «Introduction». Jostrans, 8, p. 2-4. [http:// www.jostrans.org/issue08/art_brunette.php].

Brunette, Louise, Chantal Gagnon et Jonathan Hine (2005). "The GREVIS Project: Revise or Court Calamity». Across Languages and Cultures, 6, 1, p. 29-45.

Chakhachiro, Raymond (2005). «Revision for Quality». Perspectives: Studies in Translatology, 13, 3, p. 225-238.

Comité européen de normalisation (2006). Norme européenne EN 15038:2006 F. Services de traduction - Exigences requises pour la prestation du service. Bruxelles, Institut belge de normalisation.

Defrancq, Bart et Greet Van Laecke (2009). Leesbaar schrijuen. Antwerpen, Garant; Appeldoorn, Garant.

Field, Andy (2009). Discovering Statistics Using SPSS. London, SAGE Publications Ltd.

Gile, Daniel (1995). Basic Concepts and Models for Interpreter and Translator Training. Amsterdam/Philadelphie, John Benjamins.

Graham, John D. (1989). "Checking, Revision and Editing». In C. Picken, dir. The Translator's Handbook. London, Aslib, p. 59-70.

13. Sur l'entrefaite, de nouveaux résultats, plus complets et plus poussés, ont fait l'objet de publications : Robert $(2012,2013,2014)$ et Robert et Van Waes (2014). 
Haussteiner, Ingrid (2009) «Approaches to Quality Assurance in Translation». Communication prononcée dans le cadre du $50^{\mathrm{e}}$ congrès annuel de l'American Translators Association du $28 \mathrm{au}$ 31 octobre.

Hernández Morin, Katell (2009). La révision comme clé de la gestion de la qualité des traductions en contexte professionnel. Thèse de doctorat, École doctorale Sciences humaines et sociales, Université européenne de Bretagne, Université Rennes 2, Rennes.

Horguelin, Paul A. et Louise Brunette (1998). Pratique de la révision. Brossard (Québec), Linguatech.

Horguelin, Paul A. et Michelle Pharand (2009). Pratique de la révision. $4^{e}$ éd. rev. et augm. Montréal, Linguatech.

Künzli, Alexander (2005). "What Principles Guide Translation Revision? A Combined Product and Process Study». In I. Kemble, dir. Translation Norms: What Is 'Normal' in the Translation Profession? Proceedings of the conference held on $13^{\text {th }}$ November 2004 in Portsmouth. Portsmouth, University of Portsmouth, School of Languages and Area Studies, p. 31-43.

Künzli, Alexander (2006a). «Translation Revision - A Study of the Performance of Ten Professional Translators Revising a Technical Text». In M. Gotti et S. Šarčević, dir. Insights into Specialized Translation. Bern, Peter Lang, p. 195-214.

Künzli, Alexander (2006b). «Teaching and Learning Translation Revision: Some Suggestions Based on Evidence from a Think-aloud Protocol study». In M. Garant, dir. Current Trends in Translation Teaching and Learning, Helsinki Department of Translation Studies Publication III. Helsinki, Helsinki University, p. 9-24.

Künzli, Alexander (2009). "Qualität in der Übersetzungsrevision eine empirische Studie». In H. Kalverkämper et L. Schippel, dir. Translation zwischen Text und Welt: Translationswissenschaft als historische Disziplin zwischen Moderne und Zukunft. Berlin, Frank \& Timme, p. 291-303.

Lee, Hyang (2006). «Révision: définitions et paramètres». Meta, 51, 2, p. 410-419.

Leijten,Marielle et Luuk Van Waes (2006). «Inputlog: New Perspectives on the Logging of On-Line Writing». In K.P.H. Sullivan et E. Lindgren, dir. Computer Key-Stroke Logging and Writing: Methods and Applications. Amsterdam, Elsevier, p. 73-94.

Martin, Tim (2007). «Managing Risks and Resources: a Down-toEarth View of Revision ».Jostrans, 8, p. 57-63. [http://www.jostrans. org/issue08/art_martin.php]. 
Mossop, Brian (2001). Revising and Editing for Translators. Manchester, St. Jerome.

Mossop, Brian (2007a). Revising and Editing for Translators. $2^{\text {nd }}$ ed. Manchester, St. Jerome.

Mossop, Brian (2007b). «Empirical Studies of Revision: What We Know and Need to Know». Jostrans, 8, p. 5-20. [http://www. jostrans.org/issue08/art_mossop.php].

Nord, Christiane (2005). Text Analysis in Translation: Theory, Methodology, and Didactic Application of a Model for TranslationOriented Text Analysis. Amsterdam, Rodopi.

Parra Galiano, Silvia (2005). La revisión de traducciones en la Traductologia: aproximación a la práctica de la revisión en el ámbito profesional mediante el estudio de casos y propuestas de investigación. Facultad de Traducción e Intrepretación, Universidad de Granada, Granada.

Parra Galiano, Silvia (2006). "La revisión y otros procedimientos para el aseguramiento de la calidad de la traducción en el ámbito profesional». Turjuman, 15, 2, p. 11-48.

Parra Galiano, Silvia (2007a). «La revisión como procedimiento para el aseguramiento de la calidad de la traducción: grados, tipos y modalidades de revisión». SENEZ, 32, p. 97-122.

Parra Galiano, Silvia (2007b). «Propuesta metodológica para la revisión de traducciones: principios generales y parámetros». TRANS, 11, p. 197-214.

Robert, Isabelle S. (2008). "Translation revision procedures: An explorative study». Translation and Its Others. Selected Papers of the CETRA Research Seminar in Translation Studies 2007. [http://www. arts.kuleuven.be/cetra/papers/files/robert.pdf]

Robert, Isabelle S. (2010). "Investigating the translation revision product and process through triangulating tracks: first results». Communication prononcée dans le cadre du $6^{\mathrm{e}}$ Congrès de la European Society for Translation Studies, «Treks and Tracks in Translation Studies», du 23 au 25 septembre 2010.

Robert, Isabelle S. (2012). La révision en traduction: les procédures de révision et leur impact sur le produit et le processus de révision. Thèse de doctorat, Universiteit Antwerpen, Faculteit Letteren en Wijsbegeerte, Taal- en Letterkunde.

Robert, Isabelle S. (2013). «Translation Revision: Does The Revision Procedure Matter?» In M. Bartlomiejczyk, R. Meylaerts, S. Vandepitte et C. Way, dir. Treks and Tracks in Translation Studies. Amsterdam/Philadelphie, John Benjamins, p. 87-102. 
Robert, Isabelle S. (2014). «Investigating the problem-solving strategies of revisers through triangulation. An exploratory study». Translation and Interpreting Studies, 9, 1, p. 88-108.

Robert, Isabelle S. et Luuk Van Waes (2014). «Selecting a translation revision procedure: Do common sense and statistics agree?» Perspectives, 22, 3, p. 304-320.

Rochard, Michel (1995). «Terminologie, traduction et révision: de l'expérience à la pédagogie». Communication prononcée dans le cadre du colloque La difficulté en traduction, le 30 mars 1995 à Caen (France).

Sager, Juan C. (1994). Language Engineering and Translation. Consequences of Automation. Amsterdam/Philadelphie, John Benjamins.

Segers, Winibert (2007b). «Ijkpuntenmethode». In C. Van de Poel et W. Segers, dir. Vertalingen objectief evalueren. Matrices en ijkpunten. Louvain/Voorburg, Acco, p. 92.

Shih, Claire. Y. (2006). «Revision from translators' point of view. An interview study». Target, 18, 2, p. 295-312.

Isabelle S. Robert

Universiteit Antwerpen Faculteit Letteren en Wijsbegeerte Departement Vertalers en Tolken Schilderstraat 41 2000 Antwerpen BELGIQUE isabelle.robert@uantwerpen.be 